\title{
INTEGRATION OF DATA FOR NOWCASTING OF HARMFUL ALGAL BLOOMS
}

\author{
Richard P. Stumpf $^{(1)}$, Vivi Fleming-Lehtinen ${ }^{(2)}$, Edna Granéli ${ }^{(3)}$ \\ ${ }^{(1)}$ National Oceanic and Atmospheric Administration, 1305 East-West Highway, code N/SCI1, Silver Spring MD \\ 20902, USA, Email: Richard.stumpf@noaa.gov

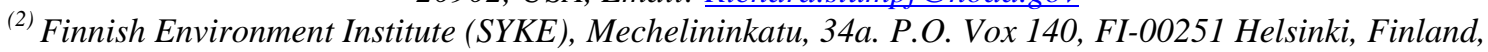 \\ Email: vivi.fleming-lehtinen@ymparisto.fi \\ ${ }^{(3)}$ Marine Sciences Centre, Linnaeus University, SE-39182 Kalmar, Sweden, Email: edna.graneli@lnu.se
}

\begin{abstract}
Harmful algal blooms (HABs) are a significant and potentially expanding problem around the world. Resource management and public health protection require sufficient information to reduce the impacts of HABs by response strategies and through warnings and advisories. To be effective, these programs can best be served by an integration of improved detection methods with both evolving monitoring systems and new communications capabilities. Data sets are typically collected from a variety of sources, these can be considered as several types: point data, such as water samples; transects, such as from shipboard continuous sampling; and synoptic, such as from satellite imagery. Generation of a field of the HAB distribution requires all of these sampling approaches. This means that the data sets need to be interpreted and analyzed with each other to create the field or distribution of the HAB. The HAB field is also a necessary input into models that forecast blooms. Several systems have developed strategies that demonstrate these approaches. These range from data sets collected at key sites, such as swimming beaches, to automated collection systems, to integration of interpreted satellite data. Improved data collection, particularly in speed and cost, will be one of the advances of the next few years. Methods to improve creation of the HAB field from the variety of data types will be necessary for routine nowcasting and forecasting of HABs.
\end{abstract}

\section{INTRODUCTION}

Problems associated with blooms of phytoplankton are global and appear to be increasing in severity and extent for reasons that are not fully understood [1]. Harmful blooms have many economic, ecological, and human health impacts. The impacts occur through alteration of marine habitats, shading, anoxia, and production of toxins or physical properties that adversely affect fish and other marine organisms [2]. While dense algal blooms can lead to anoxia, the term "harmful algal bloom" (HAB) is more often used for concentrations of algae that produce a toxin (or toxin-like) impact on organisms in the ecosystem.
Monitoring for toxic $\mathrm{HABs}$ is critical for protecting public health, wild and farmed fish and shellfish, and endangered species (such as marine mammals). For public health, monitoring requires measuring a concentration of cells or toxin in the water as well. Sampling is typically done in the area of concern at the time of year when the HAB usually occurs or after reports of discolored water or dead fish. The cost and logistics of sampling and the economic impact of delays in warning (while samples are processed) presents the need for effective detection of HABs. Data sets are collected over a few days, which means that nowcasting, an assessment (or forecast) of today's conditions made from those several days of observations, would improve the value of sampling programs by providing a more targeted identification.

The term "red tide", the common name associated with HABs, has introduced misunderstanding. Many HABs are not "red", red blooms can be non-toxic, and HABs, while often patchy, do not move like the tide. Toxic algae include several classes of microalgae, which are characterized by different colors ranging from bluegreen to golden-brown to red. These classes include diatoms, dinoflagellates, raphidophytes, cyanobacteria, prymnesiophytes, pelagophytes, and silicoflagellates [2]. Even a single type of algae can cause wide variations in watercolor with changes in concentration and changes in background scattering and dissolved absorption [3]. The more subtle problem with "red tide" involves the variety of usages. In Asia, "red tide" tends to refer to discolored water usually associated with any potentially harmful event [4]. In America, "red tide" normally refers to toxic events, including such examples like Alexandrium "blooms" in the Gulf of Maine that rarely discolor the water. Karenia brevis blooms in the Gulf of Mexico tend to produce discoloration, but the discoloration can be similar to harmless events. In many other areas, discolored water is a poor model. Many types of toxic algae are a small portion of the biomass, and so do not significantly influence the watercolor, including Alexandrium, Dinophysis, and also Pseudo-nitzschia in the northeast Pacific.

Management and public responses to $\mathrm{HABs}$ require determination of the current and potential location of the bloom. A best system has an overarching strategy that 
incorporates the various data sets, and models, that are relevant to the bloom. The data types can range from water samples to moored instrumentation to satellite data. This paper will review strategies for observing that will advance our capabilities for information necessary for nowcasting and forecasting of HABs.

\section{OBSERVATIONS}

There are four broad categories of observations: point measurements that are either single like a water sample, or continuous like a mooring; transect measurements, which are continuous in a direction; and synoptic measurements, such as imagery. Moored instruments are inherently fixed, although point measurements can be taken daily from a specific dock, which provides a form of continuity. Transects require some type of automated sampling system. Synoptic measurements would include various remote sensing systems especially including satellite measurements.

A forecast for planning may have several goals. Two broad types of concerns exist: (1) addressing the HAB at a specific area, such as the entrance to a bay or a single beach, (2) identifying the extent and severity of the HAB in order to forecast a large region. The requirements of the forecast will drive the requirements from the various types of observations and technologies used (Fig. 1).

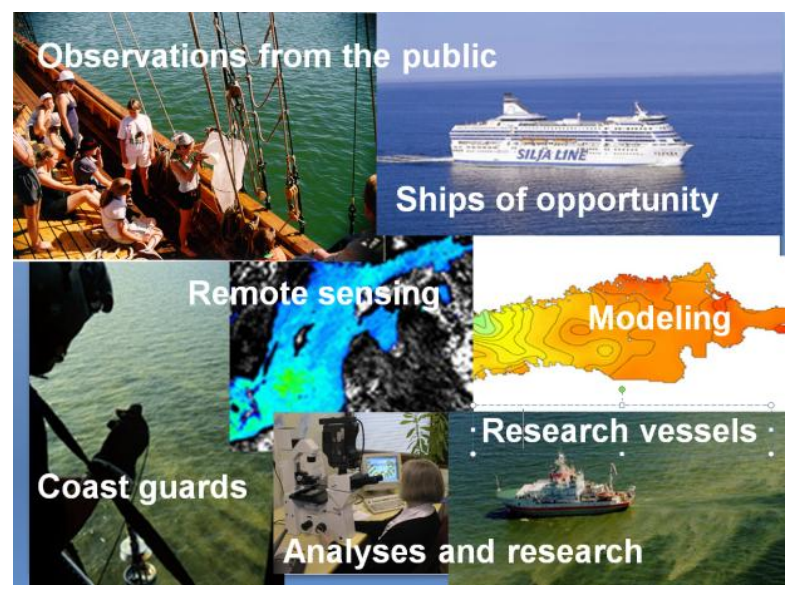

Figure 1. Types of data and analysis needed for HABs. Photos from Alg@line, Finnish Environment Institute (SYKE).

For many years, the primary tool for monitoring HABs has been point water sampling, or shellfish sampling, with subsequent microscopic or toxin analysis. This is performed either as a mobile system, where samples are collected where a bloom is reported, or as a type of fixed system, where samples are taken during the HAB season at a specific beach, shellfish bed, or fish farm. In recent years, many new tools have been brought to bear on the problem of sampling. These have significantly improved all aspects of observing. We will give examples of the types of capabilities now being used.

\section{SINGLE POINT SAMPLING}

Point sampling is the oldest scientific method, yet continues to be a vital part of observing HABs. Water samples are the most obvious form of point sampling. However, technological advances have begun to alter the acquisition of point samples. Over the last decade, a combination of technologies has appeared that has radically altered the value of point sampling, indeed of all sampling.

\subsection{Rapid Detection Methods}

Many of the advances in detection over the past decade have involved technologies that provide a simple means of detecting presence of a toxin or toxic algae. For example, there are simple antibody-based test kits that provide color changes with positive results like pregnancy tests. These require minimal training and are inexpensive to use. An example of an antibody test is the Jellett test kit [5], which indicates the presence of high levels of a toxin. A more advanced test kit is one produced by Mercury Science, Inc. that tests for several levels of domoic acid concentration [6]. This approach offers considerable value for monitoring programs by quickly identifying both samples that are above regulatory limits and those that require more precise testing. They are particularly important for identifying HABs that have no visible manifestation. With the rapid advances in biochemical detection, including methods like DNA detection (through polymerase chain reaction or PCR) it is likely that more sophisticated field kits will appear over the next ten years.

\subsection{Communications}

The most significant technological change has been in communications. Ten years ago, transmission of data and digital photographs by cell phone was quite impractical. However, the development of digital cell (and satellite) phones and the internet allow individuals to rapidly provide information to a network. This introduces a significant advantage in getting data, by placing powerful communications in the hands of individuals in the field. Continued advances in telecommunications will continue to revolutionize deployment, as individuals can now access the Internet, and thereby access digital maps that can help them plan daily sampling strategies.

By using people as part of the data collection system, an observing system can achieve rapid deployment and reconfiguration, which is not possible with moored instruments. Use of new rapid detection techniques provides a means of collecting and disseminating data rapidly through volunteer networks. Some examples of 
such systems are found in southwest Florida and on the Swedish Baltic coast.

\subsection{Beach Conditions System}

The southwest Florida coast has a risk from aerosolized brevetoxin, which can cause respiratory distress and is a specific hazard for asthmatics. To monitor this risk, the Beach Conditions system involves a professional lifeguard corps communicating with a central computer using email-enabled cell phones [7]. The phones are programmed so that the lifeguard can quickly enter reports twice daily on coughing frequency, watercolor, wind direction, relative wave height, and presence of rip currents. The reports are transmitted to a data server, so that they are automatically posted to a database and made available immediately on a Web site. What appears to be a simple capability now would have been infeasible a decade ago.

\subsection{MissAlga}

A more comprehensive approach to technology blending has occurred with the MissAlga program on the island of Öland in Sweden [8]. In 2005, the beaches were devastated when a cyanobacterial bloom covered the western Baltic Sea. Satellite imagery had been used for warnings but could not resolve the bloom sufficiently to indicate that the western side of the island was safe from the bloom, resulting in severe economic losses. More targeted resolution was needed.

The MissAlga program uses volunteers who are assigned a beach close to their home. Each morning they take a series of simple measurements. These include a digital photograph of the water, and another photo of water in a graduate cylinder next to a color scale. These are sent to a central site at the University of Kalmar. The capability allows detail that complements satellite imagery (Fig. 2). If the water color suggests a cyanobacterial bloom, a water sample is collected and placed (by the volunteer) in a $0.5 \mathrm{~cm}$ tall Utermöhl sedimentation chamber for one hour then examined at up to $400 \mathrm{X}$ magnification with an Olympus inverted microscope equipped with a digital photographic camera. Ten photographs of different parts of the chamber are taken and immediately placed on the
University website so that an expert can analyze them for the concentration of toxic Nodularia spumigena cells.

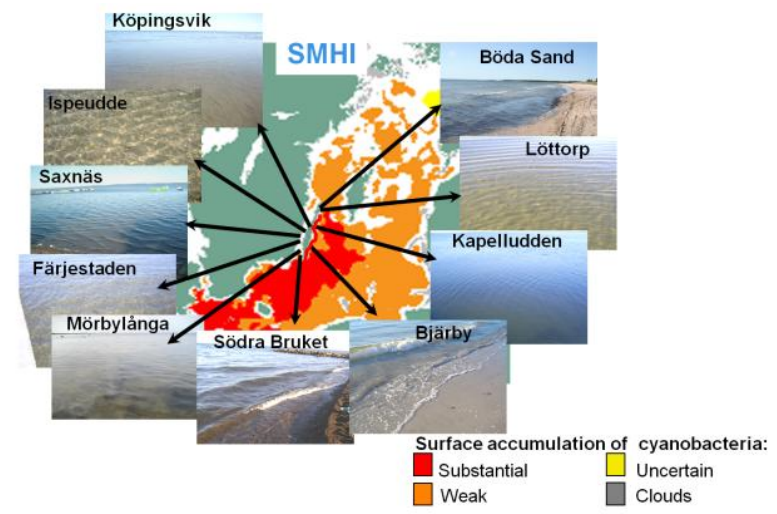

Figure 2. Imagery from SMHI in Baltic Sea on surface accumulations and volunteer pictures from Öland program taken 16 July 2006 showing differences at the beach.

The analysis of the photographs allows a determination as to whether the cell densities of $N$. spumigena are above or below the threshold where toxicity poses a risk for swimmers. The results are posted in both Swedish and English by 10 AM daily on the Linnaeus University webpage [8], at the mobile service http://mobil.hik.sel, and at a standard telephone line ( +46-480-446010). The system uses digital imaging and the internet to provide a rapid tool for assessing and quantifying both the conditions and the trends in this area (Fig. 3), without the transfer of water samples.

\section{CONTINUOUS POINT SAMPLING}

Continuous point sampling typically involves various fixed instruments. While continuous sampling, in the form of tide gauges and weather instruments have existed for decades, instruments suitable for algal blooms have appeared mostly in the past decade [9]. A significant effort has been on instrumentation that can target harmful algae. Several examples of fixed instruments are fluorescence sensors, turbidity sensors, webcams, the FlowCytobot [10], the Environmental Sample Processor (ESP) [11], and the Brevebuster [12]. 


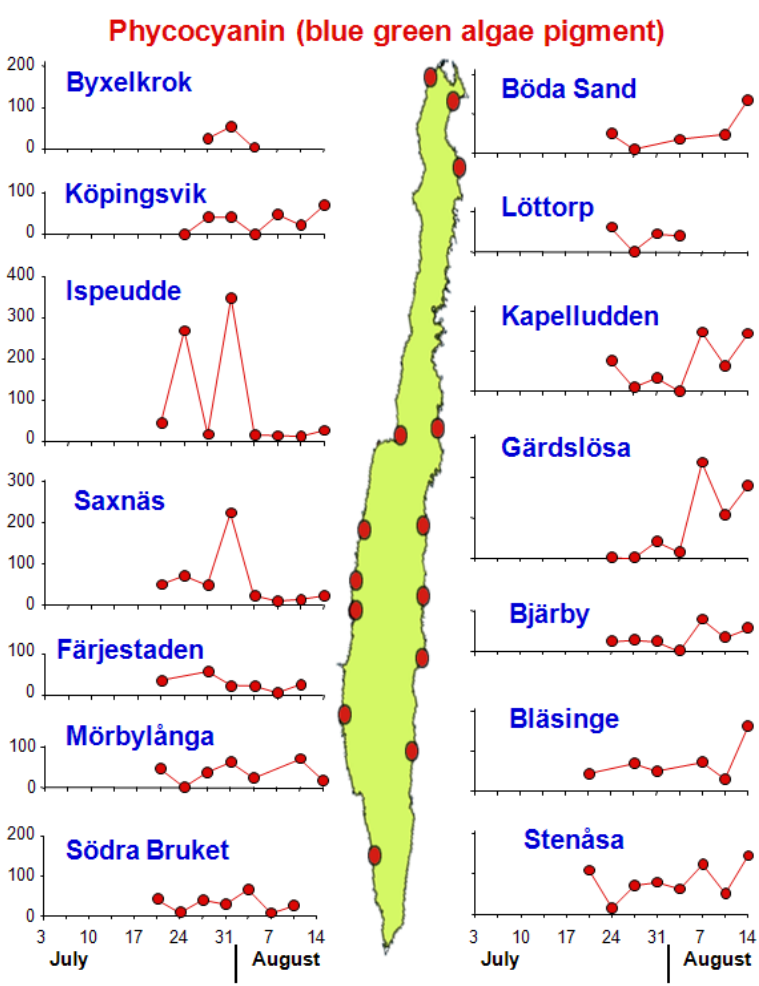

Figure 3 Time series of phycocyanin from weekly volunteer samples on Öland.

Fluorosensors provide data on fluorescence by chlorophyll and phycocyanin. Chlorophyll provides a useful indicator for intense blooms, which in some areas are harmful or toxic blooms. Phycocyanin fluorescence identifies the presence of cyanobacteria, a problem in brackish and fresh coastal water. The other sensors target specific algae.

\subsection{Brevebuster Optical Absorption}

The Brevebuster is the most mature in the sense that it has several instruments deployed along the Florida coast for several years. It determines the absorption of the water, and partitions that between dissolved and particulate components. Using a statistical similarity of the spectra to the absorption spectra of Karenia brevis (the organism that causes Florida "red tide"), the Brevebuster can determine whether Karenia dominates the absorption spectra. The Brevebuster cannot provide the precision of an ESP or FlowCytobot, but it is compact, and comparatively inexpensive to deploy. By measuring hyperspectral absorption, it is configurable to be useful for a variety of environments and biological information.

\subsection{FlowCytobot Imaging System}

The FlowCytobot uses imaging and particle analysis algorithms to identify the phytoplankton cells in the water, thereby indicating both presence and potential concentration. This allows determination of the presence of a variety of potential harmful algae, even if they are not normally in an area. The system is still in the experimental phase, but offers a definite possibility for monitoring critical areas at risk from several HABs.

\subsection{Environmental Sample Processor}

The ESP is essentially a laboratory on a mooring. It processes water samples with analyses that targets specific species through DNA and protein detection. It can be deployed on a mooring, with long-term deployments in Monterey Bay [11]. This system is well suited for a deployment at a site that systematically gets a harmful bloom of the same organism, or is a sentinel for bloom monitoring.

\section{TRANSECT SAMPLING}

For many years, research vessels have had continuous sampling systems that operate while underway. During previous decades, commercial ships have been used as platforms for routine water sampling. These so-called "ships of opportunity" have provided an excellent complement to research cruises (Fig. 4). Commercial vessels follow regular routes and timetables, while research cruises usually conduct targeted studies and include considerable additional costs for deployment. However, advances in technology have introduced new capabilities that have expanded the options in automated transect sampling.

\subsection{Autonomous Underwater Vehicles (AUVs)}

AUVs, particularly the gliders that operate passively through buoyancy, are beginning to offer new resources for sampling [13]. The gliders can operate for weeks or longer, because of the low power requirements. They can be configured with various instruments, including fluorometers and turbidity sensors. The Brevebuster has a configuration that is deployed on the Slocum Glider (Webb Research). While slow moving $\left(1 \mathrm{~km} \mathrm{~h}^{-1}\right)$ they offer three-dimensional information in a targeted area. As optical payloads become smaller, more opportunities arise, including fluorometers and backscatter sensors to enhance the hyperspectral absorption data of such instruments as the Brevebuster.

\subsection{Ships of Opportunity}

Ships of opportunity provide regular, often year-round information along routine paths on board ferries and cargo ships [14]. The routine transects are run weekly or more frequently, in some cases even twice a day. The key requirement was the development of instrument packages, either commercially produced or combined on spot that could easily be mounted on existing commercial vessels. The sampling parameters can be chosen according to preferences and may include an array of oceanographical and meteorological devices. 


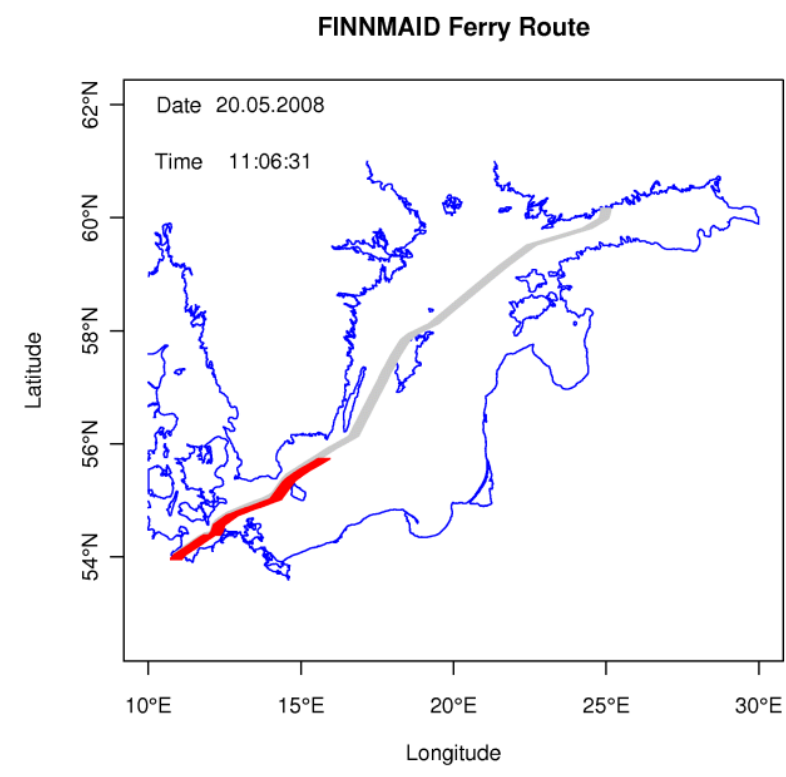

Chla fluorescence

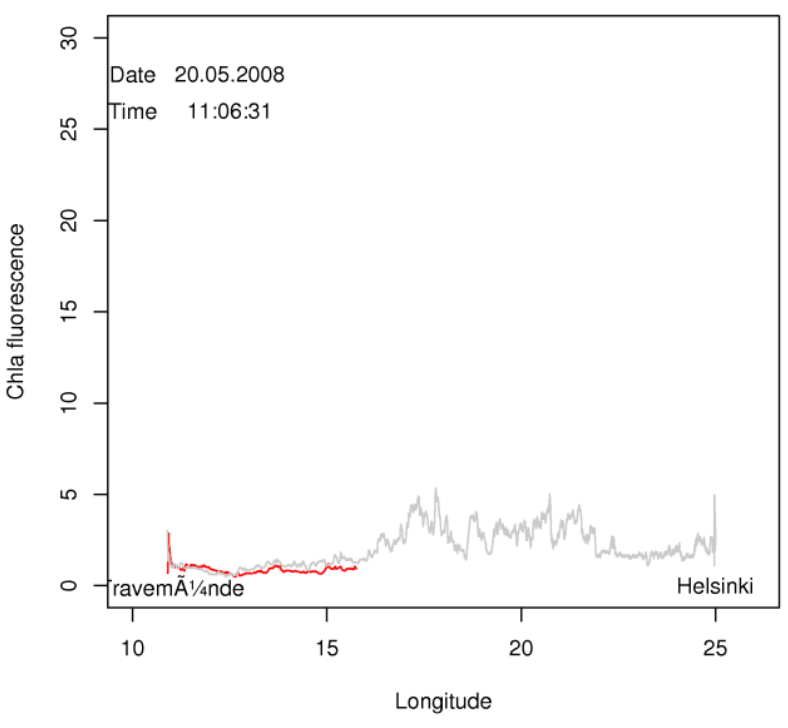

Figure 4. Top: Route taken by Ferry, red indicating location during the sampling day on return, gray indicating the previous samples. Bottom: chlorophyll data on the two transects.

An example monitoring capability is the Alg@line system in the Baltic Sea (Fig. 4), which has operated for some 15 years [16]. The system collects real-time data on routine transects, as well as weekly water samples. A specifically designed computer programme collects continuous flow-through information obtained by measurements made of surface water that is pumped through the system as the ship is running. The measured properties include chlorophyll $a$, phycocyanin fluorescence, turbidity, salinity, temperature, and nutrient concentrations. This system provides critical spatial information for monitoring of phytoplankton or cyanobacterial blooms, with data transfer in real time. Such capabilities may also provide data for validating satellite data [17].

\section{SYNOPTIC SAMPLING-REMOTE SENSING}

Remote sensing has been used to locate blooms since it was first noted that HABs in some areas discolor the water. While satellites designed for ocean color are critical for most applications, even weather satellites have provided useful monitoring capabilities for some extremely dense $\mathrm{HABs}$, such as the cyanobacterial blooms in the Baltic [18], and high biomass blooms in some estuaries [19].

The first ocean color satellite, the Coastal Zone Color Scanner (CZCS), which operated between 1978 and 1986, could not be accessed and processed in real time. However, it served a critical role in showing that ocean color could be useful for some HABs. One of its earliest images, taken only a month after launch, included a large $K$. brevis bloom in the Gulf of Mexico [20].

The significant change for remote sensing came with the launch of satellites designed for routine monitoring of ocean color, starting with SeaWiFS (the Sea-viewing Wide Field-of-view Sensor) in 1997. In 2002, SeaWiFS was joined by the Moderate Resolution Imaging Spectroradiometer (MODIS) sensor on the Aqua satellite and the Medium Resolution Imaging Spectrometer (MERIS) on the ENVISAT in 2002. These sensors have a repeat frequency of 1-2 days, There have been numerous studies and analyses of the use of satellite data for locating HABs [21] and for finding cyanobacterial blooms $[21,22]$. The incorporation of data from these sensors into routine forecasting systems has been more limited. Chlorophyll, alone is insufficient to identify a $\mathrm{HAB}$, analysis and interpretation is needed [20]. In many cases, this involves incorporating data from surface systems and environmental data. Two areas where remote sensing has become part of operational forecast systems are the Gulf of Mexico [23 and 24] and the Baltic [25 and 26].

\subsection{Gulf of Mexico Satellite Imagery}

In the Gulf of Mexico, the US National Oceanic and Atmospheric Administration (NOAA) began using satellite imagery in 1999 to monitor for blooms of $K$. brevis [23]. By 2004, this capability had become more advanced, and became a critical component of a forecast system. The primary product has been an anomaly defined as the change in chlorophyll concentration from the previous 60 days (lagged 15 days), as a bloom is a temporal change in chlorophyll). To identify a new bloom as a HAB, several methods are combined. The timing, location and the shape of the bloom are 
considered to address ecological considerations (Fig. 5). The precursor wind-driven circulation is also considered, as the HABs tend to following upwelling events [23]. More recently, some optical algorithms have been included to separate the blooms into those that are more or less likely to be Karenia HABs [27].

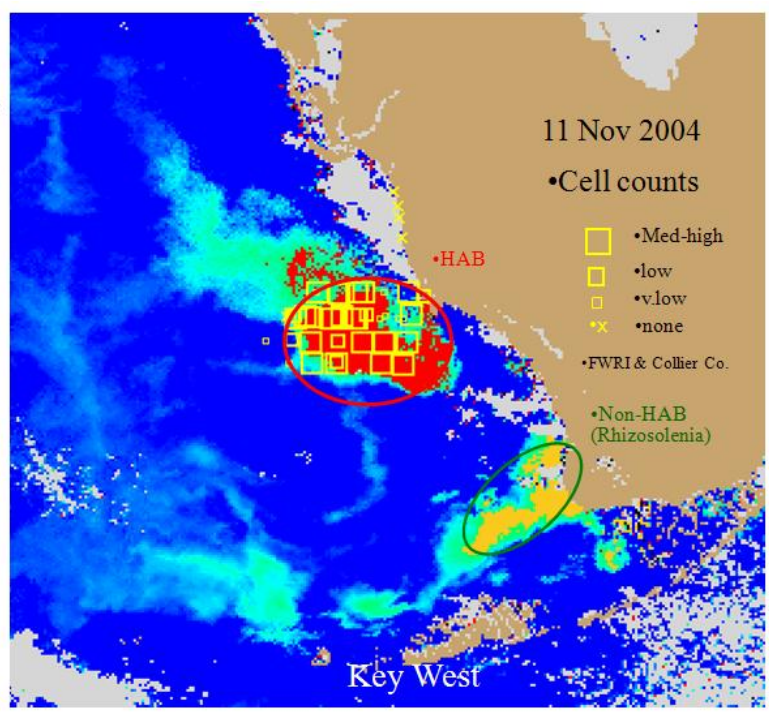

Figure 5. Overlays of satellite data and field data. Red indicates bloom edited by analyst to be flagged as a $H A B$. Yellow indicates another bloom of harmless diatom identified by previous sampling.

\subsection{Baltic Satellite Imagery}

In the Baltic Sea, satellite imagery has been used for a decade to quantify cyanobacterial blooms [18]. These blooms tend to be quite intense and concentrate near the surface, if not actually producing foams. There are several systems in place that use satellite data for these blooms. The Finish Environment Institute (SYKE) and the Swedish Meteorological and Hydrological Institute (SMHI), for example, provide daily images via the Internet. SYKE uses ENVISAT MERIS (from the European Space Agency) to interpret the weekly mean concentration of chlorophyll $a$ and to produce daily algal bloom products [25]. To produce an algal bloom product, SMHI uses the AVHRR, and occasionally the MODIS instrument [26].

\section{DATA INTEGRATION}

The ability to assess a HAB depends on our ability to determine the HAB field, i.e., the distribution and intensity of the bloom. No one data set is sufficient for this purpose. Remote sensing is often viewed as a solution to the problem, but imagery faces several problems: clouds, spatial and temporal resolution, visibility of the $\mathrm{HAB}$, and algorithm accuracy. It is rare to have even one perfectly clear image in a week. In many areas, there can be many consecutive days of complete overcast, requiring analysis of several images. Several "pixels" are needed to identify a HAB, and HABs cannot be reliably identified within two pixels of the shore, which equates to 0.6 to $2 \mathrm{~km}$ with current suitable satellite-based sensors. Assuming a HAB can be detected from satellite, there are many uncertainties in the reliability of the algorithms [27]. Satellites provide features and some algorithms can provide a good indication of the likely presence of the HAB. However, additional information is usually needed to confirm whether the feature is a HAB. The solution to these is data integration. We present examples of data integration being carried out in the Gulf of Mexico and in the Alg@line system.

\subsection{Gulf of Mexico Data Integration}

The data integration is done by analysts in the Gulf of Mexico [24]. All the relevant data is made available to the analysts in geographic form so that they can be overlain in a Geographic Information System (GIS). The analysis is made two times per week. This includes processed satellite imagery from several days, cell counts from water samples over the past ten days, reports of respiratory irritation from lifeguard stations (again over several days), and other data as available from gliders and fixed instruments. For an ongoing bloom, the analyst starts with the previous reported position. This is considered against the current satellite imagery. The positions are adjusted based on the other data sets, and finally a distribution along the coast is determined.

\subsection{AIg@line Data Integration}

An analysis approach is also made for the Alg@line project in SYKE [16]. In this area, the emphasis is on providing information on the day-to-day cyanobacterial bloom situation for the public. The data sets - including visual observations from educated volunteers and coast guard pilots, as well as remote sensing and ship-ofopportunity data-are transferred to a four-step scale ranging from "not observed" to "very abundant". The result is combined in a single map (Fig. 6). The scale was originally developed to describe visual observations made by educated volunteers and coast guard pilots along the Finnish coast. The phycocyanin observations collected on ships-of-opportunity are scaled with the help of microscopic analysis of the cyanobacteria concentration achieved from the regular (weekly) water samples. The information acquired by remote sensing, originally expressed as bloom probabilities, is transformed through comparison with the existing observations from the coast guard. These data sets are 
merged into GIS environment in order to provide a map of the spatial distribution and relative intensities of the blooms.

\section{MERGING DATA AND TRANSPORT MODELS}

In the modelling environment, the concern is often on the circulation model. Modern circulation models have the ability to assimilate a variety of physical data types [28], so that they can produce reasonable current fields.

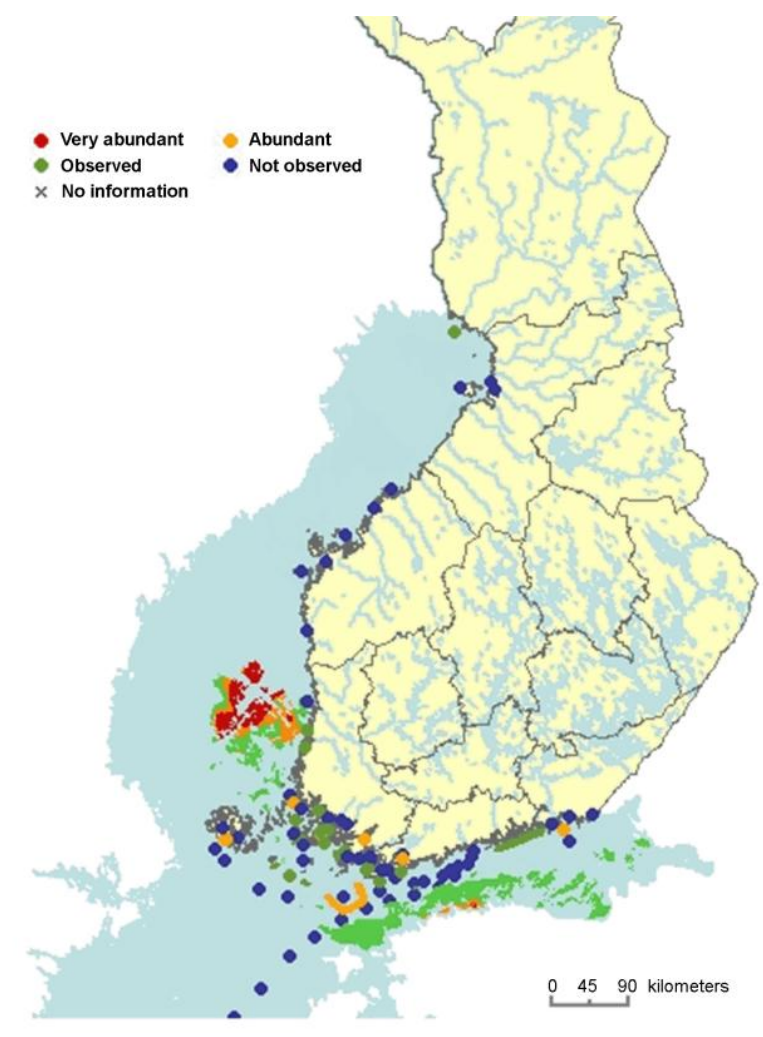

Figure 6. Data from different sources integrated into Alg@line analysis of blooms. (Base map data sets sources $($ S SYKE and regional environment centers (coastline@MML); (C) Affecto Finland Oy Karttakeskus, Permit L4659; and () National Land Survey of Finland, Permit 7/MML/09.)

For nowcasts, an additional option is the use of surface currents determined by high frequency (HF) radar [28]. $\mathrm{HF}$ radar can produce surface current fields to over 100 $\mathrm{km}$ offshore.

Of course, the other necessary component in any model is the HAB field used to initialize the model. In most cases, creating this field requires the type of integration described in the previous section. Some other types of initialization exist for certain types of HABs.

In the Gulf of Maine, Alexandrium fundyense, which produces annual toxic blooms, forms a resting stage called a cyst in order to over-winter [29]. The initialization for a model is a map of cyst abundance in the bottom sediments (Fig. 7). The cells re-emerge based on temperature and season. This map is used to initialize a coupled model of population dynamics with a fully three-dimensional hydro-dynamic model, resulting in modelled cell distributions through the season [30].

To maintain accuracy, the HAB field would need to be assimilated regularly into a model. Much less work has been done in this area. One option is to convert the HAB field to particles, then using a particle-tracking tool to move them with the modelled currents. An experimental system for Lake Erie, USA, has used this approach (Fig. 8). The HAB can be identified and quantified by MERIS [31]. It is converted to particles, with the density based on the concentration. After the particles are moved, the field is converted back to concentrations. While the satellite imagery provides the potential of a continuous field that could allow for automated assimilation, this does not always work in practice. Gaps appear as a result of clouds and features are flagged that are identified from the analysis as being something other than the HAB. To establish a robust continuous field for assimilation, manual analysis is required.

\section{SKILL ASSESSMENT}

Any systems for nowcasts or modelling will require an assessment of skill. Skill assessment provides a level of confidence in the distributions but it also identifies areas that require improvement. Skill assessment has been performed for the Gulf of Maine experimental forecasts [32] and is performed routinely as part of the Gulf of Mexico operational forecasts [22 and 33]. In the Gulf of Maine, the assessment evaluated the spatial confidence of the forecast. The model, which runs over several months each year, described over $50 \%$ of the variance at scales of greater than $50 \mathrm{~km}$ [32]. The skill assessment also identified areas where the model over-estimated currents, thereby causing more rapid transport, and in some areas, the model over-estimated the cell concentration. These results suggest the potential value of assimilation of HAB data while the model is running, potentially altering the concentrations or fields to avoid these errors.

In the Gulf of Mexico, respiratory irritation is one of the key forecasts [22 and 33]. This forecast is evaluated with lifeguard reports of the impact [7]. The HAB field cannot be determined to finer scale than $30-60 \mathrm{~km}$, about the length of a county, so the forecast is made at that scale. The assessment found that these forecasts were accurate over $70 \%$ of the time. However, at individual beaches, the countywide forecast was accurate only $20 \%$ of the time. The problem is that the blooms are patchy at a finer scale than current sampling can resolve. This result identifies a need for increased resolution in the $\mathrm{HAB}$ field used for nowcasts. 


\section{FUTURE NEEDS AND PROSPECTS}

The observational capabilities are rapidly increasing. There is no one system or technology that can best provide the necessary data. Much finer resolution will significantly enhance nowcasting and ultimately forecasting. That means more data must be acquired, which in turn identifies a critical need for rapid detection techniques, together with effective use of communities and organizations that live near or regularly use the water.
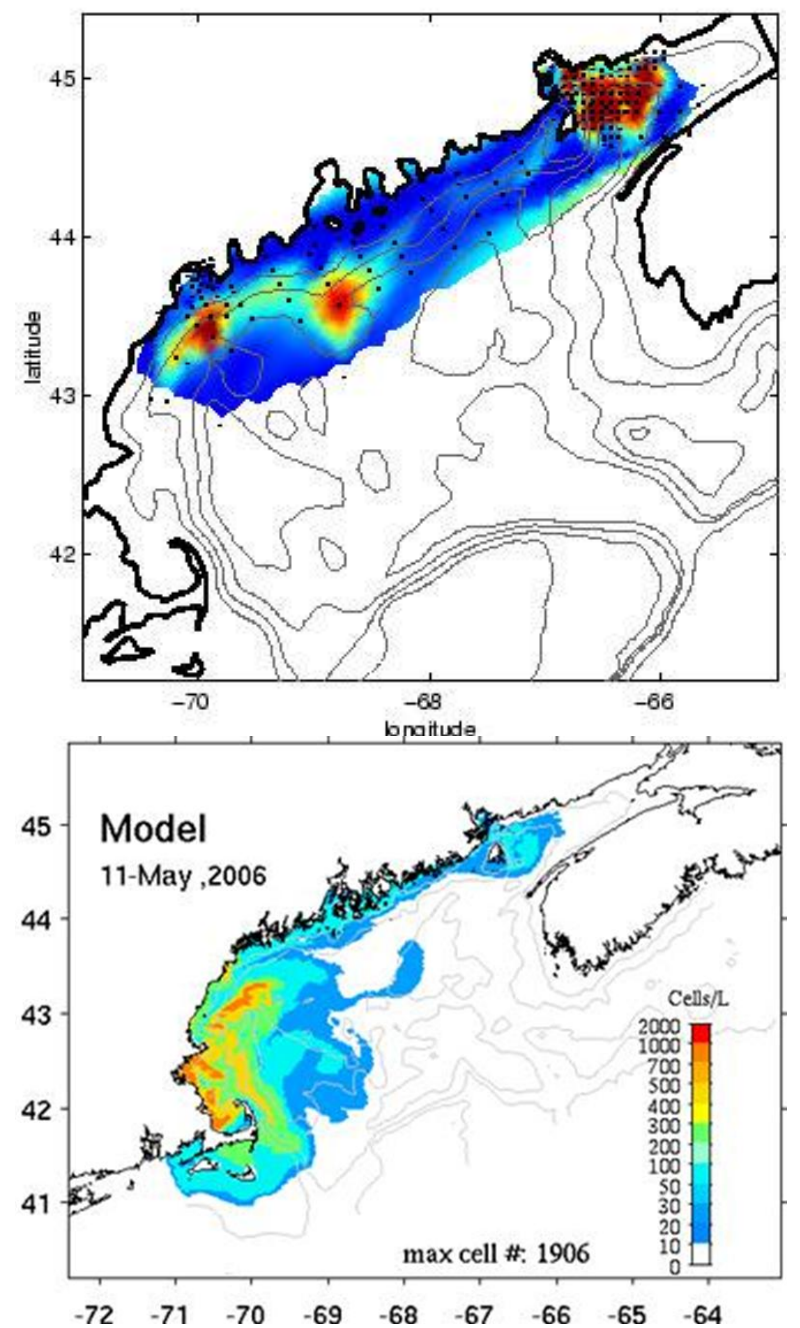

Figure 7. Top: cystmap made from samples to initialize the Gulf of Maine Alexandrium model. Bottom: Modeled cell distribution during the bloom season.

A critical concern is the implementation of data collection through routine transects. This will involve continued enhancements of systems for commercial vessels (such as through the FerryBox) and by expanded use of AUV gliders. Routine deployments of underwater vehicles (or AUVs) are now appearing [13], and one of these can cost about the same as a moored instrumentation system. AUVs offer the necessary three-dimensional structure that cannot be achieved from most other instrumentation systems. They also have particular value in ease and rapidity of redeployment, a critical issue for blooms that do not appear in the same region. It is possible that new toxin sensors will be developed that could be installed on AUVs, giving yet another capability.

Sophisticated sensors like the ESP and FlowCytobot are much more costly, but could be ideal for sentinel sites, whether at inlets or at key points in coastal currents. For example, the FlowCytobot deployed at the inlet to a major oyster-producing bay in Texas identified toxic Dinophysis entering the bay just a few weeks before a major seafood festival [35]. Dinophysis had never occurred at toxic levels in the Gulf of Mexico, and this warning averted a potential health disasters. These types of sensors should be considered a priority in configuring moorings in key sites to complement the higher resolution "manual" sampling.

While engineering solutions are often sought, manual data collection will become more powerful as measurement tools become cheaper and simpler to use. In the United State, a sophisticated volunteer program has evolved, the Phytoplankton Monitoring Network [34]. This program provides quality microscopes (with digital imaging) to volunteers, with the training to identify the dominant HABs. Such a capability could easily be envisioned to be combined with the use of other instruments or measurement methods, such as the antibody test kits.

We can envision enhancing a system like MissAlga with identification algorithms, such as found in the FlowCytobot system. In addition, trained volunteers could analyze water in the field with new test kits and transmit those results to the central server. Key water samples could be delivered to a site having sophisticated identification systems, such as advanced antibody analyses (enzyme labelled immune-sorbent assay or ELISA) or DNA analysis (polymerase chain reaction or PCR). The framework of this strategy is being demonstrated now through the MissAlga system [8], system, the PMN [33], the Beach Conditions system [7].

Remote sensing has the potential to see significant advances with the continued development of unmanned aerial vehicles. The significant limitation in satellite remote sensing is resolution within one kilometre of the coast. Also, hyperspectral data collected remotely offers the potential to better discriminate phytoplankton communities [36]. Lidar also has been demonstrated as a detection capability from standard aircraft [36]. We are at least a decade from a satellite that can offer hyperspectral data with the necessary spatial and temporal resolutions (300 $\mathrm{m}$ each day). However, installing hyperspectral instruments or lidars on UAVs offers the potential for rapid inexpensive monitoring. 
The modelling community has achieved sophisticated and detailed circulation models. The limitation, though, is on the assimilation of HAB data or other phytoplankton data into models [38]. Simply introducing a point sample will not address the public health concerns. A field will be required for the models. Simplifying data integration will be the next critical area of research and advancement. This type of integration should take into account data collected on different days and from different sources [38]. Ultimately, the analysis will need to incorporate transport of observations made on different days. This would include movement of analyzed fields obtained from satellite, keeping in mind
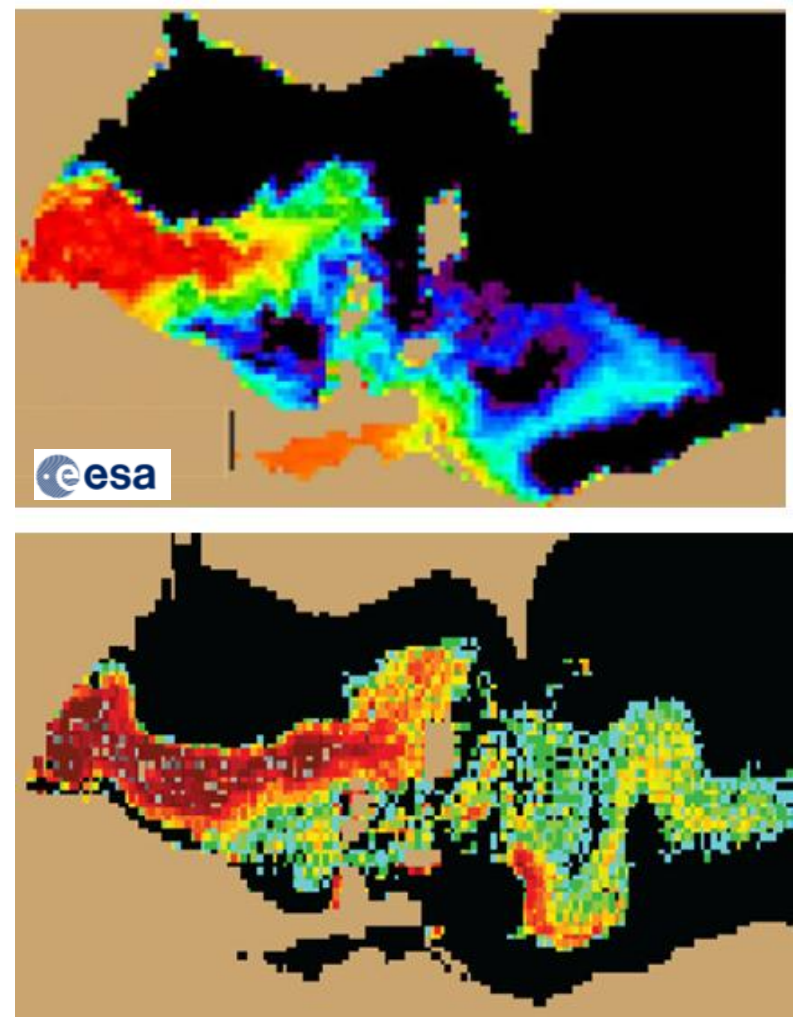

Figure 8. Top: Cyanobacteria estimated with MERIS for western Lake Erie, USA. Bottom: modelled distribution to 3 days later.

that multiple observations and images may be required to see the entire field. At this time, it is difficult to envision development of robust HAB fields without human analysis. However, providing an integrated field could standardize and speed the analysis, thereby providing the resolution so that assimilation into models leads to accurate and usable forecasts.

Information on $\mathrm{HABs}$ is starting to move to managers and the public in more useful forms. Online access to data for point locations is demonstrated using simple online mapping tools, such as Google Maps (e.g. the Florida Beach Conditions report [7]). Fields of data and imagery will need to be distributed in standard GIS formats rather than as pictures. Immediate dissemination of analyzed products in usable data formats (geoTIFF, GIS shape files, etc.) will provide the critical data needed by the various communities. Such information must include analysis as part of the system and metadata, as well as training and interpretative documents [39]. Providing analysis and education will increase the value and usability of the observations of these threats.

In order to examine concerns of human or climate impacts on development of HABs, significantly better information is needed on the distribution of HABs. For cyanobacterial blooms in the Baltic, satellite has been sufficient to identify quantity and aerial distribution to permit assessment of inter-annual variability [15]. For most other areas, the data sets consist of only toxin levels at the coast that were collected for seafood safety, this means that the same type of observing strategies needed for improved nowcasts are needed for climate [40]. As many significant blooms develop offshore and are dependent on factors such as wind driven-circulation to reach the coast [41], coastal data has limited value in assessing climate patterns. Making these evaluations will be another application that requires determination of the HAB field from an integration of all systems of HAB observations.

\section{ACKNOWLEDGMENTS}

Many thanks to Dennis McGillicuddy of Woods Hole Oceanographic Institution and Seppo Kaitala and Paula Väänänen of the Finnish Environment Institute (SYKE) for figures and discussion. References to any nongovernment product or service do not constitute an endorsement by the U.S. National Oceanic and Atmospheric Administration.

\section{REFERENCES}

1. Glibert, P.M and Co-Authors. (2005). The global, complex, phenomena of harmful algal blooms. Oceanography $18(2), 132-141$.

2. Landsberg, J.H. (2002). The effects of harmful algal blooms on aquatic organisms. Reviews in Fisheries Science 10(2), 113-390.

3. Dierrsen, H.M., Kudela, R.M., Ryan, J.P., Zimmeran, R.C. (2006). Red and black tides: quantitative analysis of water-leaving radiance and perceived color for phytoplankton, colored dissolved organic matter, and suspended sediments. Limnology and Oceanography, 51(6), 2646-2659.

4. Ishizaka, J.Y. and Co-Authors. (2006). Satellite detection of red tide in Ariake Sound, 1998-2001. Journal of Oceanography. 62, 37-45.

5. Laycock, M.V., Jellett, J.F., Easy, D.J., Donovan, M.A. (2006). First report of a new rapid assay for diarrhetic shellfish poisoning toxins. Harmful Algae, 5, 74-78. 
6. Litaker, R.W. and Co-Authors (2008). Rapid enzymelinked immunosorbent assay for detection of the algal toxin domoic acid. Journal of Shellfish Research, 27(5):1301-1310.

7. Kirkpatrick, B. and Co-Authors (2008). Florida red tide and human health: a pilot beach conditions report system to minimize human exposure. Science of the Total Environment, 402, 1-8.

8. Miss Alga, http://www.hik.se/alg/default.aspx

9. Babin, M and Co-Authors (2005). New approaches and technologies for observing harmful algal blooms. Oceanography 18(2):211-227.

10. Olson, R.J., Shalapyonok, A., Sosik, H.M. (2003). An automated submersible flow cytometer for analyzing pico- and nanophytoplankton: FlowCytobot. Deep-Sea Research I, 50, 301-315.

11. Greenfield, D.I. and Co-Authors (2008). Field applications of the second-generation Environmental Sample Processor (ESP) for remote detection of harmful algae: 2006-2007. Limnology and Oceanography: methods (6), 667-679.

12. Schofield, O., Bosch, J., Glenn, S. M., Kirkpatrick, G., Kerfoot, J., Moline, M., Oliver, M., Bissett, W. P. (in press). Harmful algal blooms in a dynamic environment: How can optics help the field-going and sample poor biologist? In Real Time Coastal Observing systems for ecosystems dynamics and harmful algal blooms. Babin, M. And Cullen, J. J. (Eds) UNESCO, Paris.

13. Testor, P. \& Co-Authors (2010). "Gliders as a Component of Future Observing Systems" in these proceedings (Vol. 2), doi:10.5270/OceanObs09.cwp.89

14. Leppänen, J.-M., Rantajärvi, E., Hällfors, S., Kruskopf, M. and Laine, V. (1995). Unattended monitoring of potentially toxic phytoplankton species in the Baltic Sea in 1993. Journal of Plankton Research 17(4):891-902.15. FerryBox. http://www.ferrybox.org

16. Alga-line. http://www.fimr.fi/en

17. Vepsäläinen, J and Co-Authors ( 2003). The combined use of optical remote sensing data and unattended flowthrough fluorometer measurements in the Baltic Sea. International Journal of Remote Sensing 26(2):261-282.

18. Kahru, M., Savchuk, O.P., Elmgren, R. (2007) Satellite measurements of cyanobacterial bloom frequency in the Baltic Sea: interannual and spatial variability. Marine Ecology Progress Series 343, 15-23.

19. Stumpf, R.P. and Tyler M.A. (1988). Satellite detection of bloom and pigment distribution in estuaries. Remote Sensing of Environment 24: 385-404.

20. Steidinger, K.A. and Haddad, K. (1981). Biologic and hydrographic aspects of red tides. Bioscience. 31, 814819

21. Stumpf, R.P. and Tomlinson, M.C. (2005). Remote sensing of harmful algal blooms, IN: Remote Sensing of Coastal Aquatic Environments: Technologies, Techniques and Applications. R. Miller, C. Del Castillo, B. McKee (eds.), Kluwer Academic Publishers.
22. Kutser, T. (2009). Passive optical remote sensing of cyanobacteria blooms in coastal and inland waters. International Journal of Remote Sensing. 30(17), 30693085 .

23. Stumpf, R.P. and Co-Authors (2003). Monitoring Karenia brevis blooms in the Gulf of Mexico using satellite ocean color imagery and other data. Harmful Algae 2, 147-160

24. Fisher, K.M and Co-Authors (2006). Annual Report of the Gulf of Mexico Operational Harmful Algal Bloom Forecast System. NOAA Technical Report NOS COOPS 047. Silver Spring, Maryland, USA.

25. SMHI, http://www.smhi.se/en/theme/monitoring-algaefrom-satellite-1.11923

26. Environment Finland ,http://www.environment.fi/default.asp?contentid=87506 \&lan=en

27. Tomlinson, M.C., Stumpf, R.P., Wynne, T.T. (2009). An evaluation of remote sensing techniques for enhanced detection of the toxic dinoflagellate, Karenia brevis, in the Gulf of Mexico, Remote Sensing of Environment, 113:598-609.

28. Beegle-Krause, C. \& Co-Authors (2010). "Observations as Assets in Decision Support" in these proceedings (Vol. 2), doi:10.5270/OceanObs09.cwp.03

29. Anderson, D.M, and Co-Authors (2005). Alexandrium funyense cyst dynamics in the Gulf of Maine. Deep-Sea Research II 52(19-21), 2522-2542.

30. McGillicuddy, D.J., Jr., Anderson, D.M., Lynch. D.R., Townsend, D.W. (2005). Mechanisms regulating largescale seasonal fluctuations in Alexandrium fundyense populations in the Gulf of Maine. Deep-Sea Research II 52(19-21), 2698-2714.

31. Wynne, T.T., and Co-Authors (2008). Relating spectral shape to cyanobacterial blooms in the Laurentian Great Lakes. International Journal of Remote Sensing, 29(12): 3665-3672.

32. Stock, C.A. McGillicuddy, D.J., Jr., Solow, A.R., Anderson, D.M. (2005). Evaluating hypotheses for the initiation and development of Alexandrium fundyense blooms in the western Gulf of Maine using a coupled physical-biological model. Deep-Sea Research II, 52, 2715-2744

33. Stumpf, R.P., Tomlinson, M.C., Calkins, J.A., Kirkpatrick, B., Nierenberg, K., Currier, R., Wynne, T.T (2009). Skill Assessment for an operational algal bloom forecast system. (special issue on Skill Assessment of Ecological Oceanographic Models) Journal of Marine Systems, 76:151-161.

34. NOAA Phytoplankton Monitoring Network, http://www.chbr.noaa.gov/pmn/

35. Oceanus http://www.whoi.edu/oceanus/viewArticle.do?id=46486

36. Craig, S.E. and Co-Authors (2006), Use of hyperspectral remote sensing reflectance for detection and assessment of the harmful alga, Karenia brevis. Applied Optics 45: 5414-5425. 
37. Wright, C.W. and Co-Authors (2001). Next-generation NASA Airborne Oceanographic Lidar System. Applied Optics 40(3): 336-342.

38. Gregg, W.W and Co-Authors (2009). Skill assessment in ocean biological data assimilation. Journal of Marine Systems 76: 16-33.

39. Fleming, L.E. and Co-Authors (2007). Evaluation of harmful algal bloom outreach activities. Marine Drugs 5:208-219.

40. Moore, S. and Co-Authors (2009). Recent trends in paralytic shellfish toxins in Puget Sound, relationships to climate and capability for prediction of toxic events. Harmful Algae 8:463-477.

41. Li, Y. and Co-Authors (2009). Investigation of the 2006 Alexandrium fundyense bloom in the Gulf of Maine: Insitu observations and numerical modeling. Continental Shelf Research 29:2069-2082. 